\title{
Temporal discrimination of alternate days in rats
}

\author{
Matthew J. Pizzo \\ University of York, York, England \\ AND \\ Jonathon D. CRYstal \\ University of Georgia, Athens, Georgia
}

\begin{abstract}
The objective was to determine whether rats could learn to time a 48 -h interval. Rats $(n=6)$ were continuously housed in operant chambers in constant darkness. The feeding cycle consisted of unlimited access to food for $6 \mathrm{~h}$, followed by $42 \mathrm{~h}$ without access to food (i.e., meals were available on alternate days, contingent on breaking a photobeam in the food trough). Head entries into the trough increased as a function of time prior to the meal; this increase was higher, relative to the increase that occurred at the same time of day on alternate (i.e., nonfood) days. These data suggest that rats discriminated alternate days. Next, two meals were omitted to dissociate mechanisms of a self-sustained endogenous rhythm, interval timing, and alternation. Response rate increased periodically every $24 \mathrm{~h}$, which suggests that the rats anticipated alternate days by discriminating the status of the previous day as a meal or a nonmeal day.
\end{abstract}

Learning the time at which events occur has long been known to be important for adaptation. For animals in the wild, timing is important for acquiring necessary resources. Research has provided numerous examples illustrating animals' knowledge of when food is available, both in captivity (Bolles \& Moot, 1973; Mistlberger, de Groot, Bossert, \& Marchant, 1996; Richter, 1922; Stephan, Swann, \& Sisk, 1979; White \& Timberlake, 1995) and in the wild (Daan \& Koene, 1981) during mating (Silver \& Bittman, 1984) as well as when prey is available (Rijnsdorp, Daan, \& Dijkstra, 1981). Much research in this area has shown that animals learn when daily events occur. For example, honeybees have been known to visit the breakfast table when food is regularly taken there (Forel, 1910). In the laboratory, rats have been known to anticipate a daily meal to a high degree of accuracy (Crystal, 2001).

However, many events that take place in the environment occur less frequently than every day. It would be useful for animals to learn how to acquire resources that transpire with atypical patterns (i.e., multiple times within a day or less often than daily). Behavior observed under such patterns has coincided with the lunar cycle and with seasonal and annual periods (Aschoff, 1981). Of course, daily, monthly, seasonal, and annual periods are characterized by large gaps. Moreover, there are few instances in the literature to suggest that animals note the time of an event within this gap (i.e., occurring more often than each month but less frequently than every day).

The availability of food would be one potential irregularly occurring event that animals could need to learn. Gallistel (1990) reported an unpublished work by
Caubisens and Edmonds, describing an experiment that involved feeding birds at 0800 every other day (i.e., an interfood interval of $48 \mathrm{~h}$ ). In that study, a feeder was stocked with food on alternate days. Thereafter, the birds learned to restrict their visits to the feeder to the times at which food was available. Occasionally, an experimenter arrived earlier than the regular feeding time to find that the birds were not present. Whenever the researcher arrived late, the birds were found waiting for food. In order to investigate whether the experimenter's presence at the feeder induced the birds to visit, random visits at other times were conducted, which resulted in no bird visits. Other evidence that animals can learn a daily alternation pattern exists (Capaldi \& Lynch, 1966; Capaldi \& Spivey, 1964), but the mechanism is unknown.

There are three potential mechanisms that may be responsible for the temporal performance observed in the Caubisens and Edmonds (Gallistel, 1990) study. First, timing may be based on the use of an oscillator. Gallistel proposed that temporal performance is based on the use of multiple oscillators. The best-studied oscillatory system is the circadian-timing system (Aschoff, 1981; Takahashi, Turek, \& Moore, 2001). Gallistel proposed that shorter and longer period oscillators are used to represent the time of occurrence of individual events. According to this proposal, intervals are computed by subtracting the times of occurrence for two events, and associations between events are based on correlation patterns in the times of occurrence. Within this framework, discrimination of alternate days may be based on an infradian oscillator (e.g., an oscillator with a period of $48 \mathrm{~h}$ ).

J. D. Crystal, jcrystal@uga.edu 
A second potential mechanism is interval timing. According to this proposal, elapsed time is represented by the number of pulses accumulated since the start of a timing episode (Gibbon, 1991). For example, when a meal begins, the accumulation process may be reset; consequently, at the start of each meal, the number of pulses accumulated is a representation of the interval since the last reset of the system. Henderson, Hurly, Bateson, and Healy (2006) trained hummingbirds to obtain food from eight artificial flowers, four that were replenished $10 \mathrm{~min}$ after being emptied and four that were refilled $20 \mathrm{~min}$ after being emptied. The data revealed that the birds visited the 10-min flowers earlier than they did the 20-min flowers. Flower visits matched the refilling schedules. Obtaining a reward under such conditions was likely accomplished with the use of an interval-timing mechanism (Crystal, 2006).

A third potential mechanism is alternation. For example, an animal may learn that food is available on alternate days by alternating its behavior with respect to the presence/absence of food. According to this hypothesis, when an animal encounters food on a given day, it may detect that food was not available on the preceding day. In contrast, on those days on which food is unavailable, it may detect that food was present on the previous day. This strategy amounts to making a conditional discrimination that is based on the status of the previous day (i.e., meal or nonmeal). A circadian oscillator would provide information about time of day, and we propose that the alternation mechanism discriminates alternate days. Pizzo and Crystal (2004) have documented the use of an alternation strategy in a time-place discrimination task using the T-maze (i.e., the rats alternated between the morning and the afternoon reward locations).

The three potential mechanisms described above would make the same prediction regarding the Caubisens and Edmonds (Gallistel, 1990) study (i.e., when meals are presented on alternate days, periodic behavior is predicted by oscillator, interval, and alternation mechanisms). However, to dissociate these mechanisms, it is necessary to discontinue the delivery of meals. A defining feature of a circadian oscillator is that periodic output continues after the termination of periodic input. Indeed, an important piece of evidence in support of an oscillator mechanism comes from studies that suggest that the anticipation of the meal continues for more than one cycle when multiple meals are omitted (Boulos, Rosenwasser, \& Terman, 1980; Escobar, Díaz-Muñoz, Encinas, \& AguilarRoblero, 1998). Because an oscillator is endogenous and self-sustaining, behavior generated by a 48 -h oscillator is expected to continue after periodic input terminates.

By contrast, a hallmark feature of an interval clock is that it estimates the elapsed time between the presentation of salient events, as in the case of a stopwatch (Church, 1978). Consequently a defining feature of an intervaltiming system is that it is periodic only if presented with periodic input (Gibbon, Fairhurst, \& Goldberg, 1997). Periodic output from an interval-timing system is expected to cease if the periodic input is discontinued.
Finally, an alternation mechanism produces anticipation of the meal after the occurrence of each nonmeal. With a 48-h interfood interval, the nonmeal event occurs on alternate days. If the delivery of meals is discontinued, each test day occurs after a nonmeal event. Consequently, the termination of meals should produce anticipatory behavior on each subsequent test day.

The present experiment was designed to investigate whether rats can learn a 48-h pattern of food availability and to evaluate the mechanism. On alternate days, each food pellet in a 6-h meal was delivered contingent on interruption of a photobeam inside the food trough. If the rats could discriminate between food days and nonfood days, the response rate in anticipation of the meal should be higher than the rate observed $24 \mathrm{~h}$ earlier. However, these potential results would not identify the mechanism. In order to test for the presence of a self-sustaining oscillator, it is necessary to deprive the rats for a period that is equivalent to two interfood interval cycles (i.e., for $96 \mathrm{~h}$ ). A 48-h oscillator predicts that behavior would be periodic with a period of $48 \mathrm{~h}$. An interval-timing mechanism predicts anticipation of the first 48-h interval but that no additional timing will ensue (i.e., not reset by a previous meal). An alternation mechanism predicts anticipation of the first 48-h interval, followed by anticipation of subsequent $24-\mathrm{h}$ intervals (i.e., treating each previous day as a nonfood event).

\section{METHOD}

\section{Subjects}

Male Sprague-Dawley rats $(n=6)$, Rattus norvegicus (Charles River), were used in the study. All the rats were randomly assigned to an operant chamber. The rats were 97 days old and weighed $286 \mathrm{~g}$ at the beginning of the experiment. They were housed in constant darkness within the testing chambers for the entire length of the study. Dim red lights were present in the testing rooms at all times. Food pellets (Noyes Precision pellets, Improved Formula A/I; Research Diets, New Brunswick, NJ) were used for reinforcement. Water was available at all times.

\section{Apparatus}

Each rat was tested in an operant chamber $(30.5 \times 24.1 \times 29.2 \mathrm{~cm}$; Med Associates, ENV-007-VP, Georgia, VT). These chambers were equipped with two retractable levers (ENV-112CMX, $4.8 \times 1.9 \mathrm{~cm}$ ) that are $6.4 \mathrm{~cm}$ above the floor. The floor of the chamber consisted of 19 stainless steel rods $(4.8-\mathrm{mm}$ diameter, $1.6 \mathrm{~cm}$ of spacing between them) with a stainless steel waste tray positioned below it. A pellet dispenser (ENV-203IRX), capable of dispensing 45-mg pellets, was situated outside the testing chamber and connected to a food trough (ENV-200R2M). A photobeam (ENV-254) placed $1 \mathrm{~cm}$ inside the food trough $(1.5 \mathrm{~cm}$ from the trough bottom) detected head entries. A response was registered at the time that the photobeam was interrupted. The photobeam interruption had to end before the next response was registered. Therefore, the duration of photobeam breaks was not recorded. A water bottle was placed outside of the chamber, with the tube inserted across from the food trough. Each chamber included a speaker $(\mathrm{ENV}-224 \mathrm{AM}, 8.0 \times 11.0 \mathrm{~cm})$, a relay $(\mathrm{ENV}-$ $135 \mathrm{M})$, and a houselight (ENV-227M). Each chamber was housed in a sound-attenuation cabinet $(\mathrm{ENV}-016 \mathrm{M}, 66.0 \times 35.6 \times 55.9 \mathrm{~cm})$. A Celeron computer $(850 \mathrm{MHz}$ ) running Med-PC for Windows (Version 4.0), located in a nearby room, controlled experimental events and recorded the time of each event with $10-$ msec accuracy. 


\section{Procedure}

The experimental contingencies were introduced the moment the rats were placed in the operant chambers. A meal was immediately available for $6 \mathrm{~h}$, followed by no food for $42 \mathrm{~h}$. Subsequent meals began every $48 \mathrm{~h}$ (i.e., meal onset to meal onset was $48 \mathrm{~h}$ ). Unlimited access to food was available during each meal; food was obtained by interrupting a photobeam in the food trough, using a variable interval 5-sec schedule of reinforcement. Previous work has shown that food approach behavior, such as nose pokes within a food trough, is an especially sensitive measure of anticipatory behavior (Aragona, Curtis, Davidson, Wang, \& Stephan, 2002). Training consisted of 56 days ( 28 cycles). At the end of the 56 th day, the rats were deprived of food for $96 \mathrm{~h}$ (i.e., probe test). The rats were removed from the chamber and fed food ad lib immediately after the experiment ended. All the procedures were approved by the institutional animal care and use committee at the University of Georgia following the guidelines of the National Research Council Guide for the Care and Use of Laboratory Animals.

During the experiment, access to the chambers was restricted as follows. No access ever occurred during the $3 \mathrm{~h}$ prior to the meal. Access occurred every week at a randomly selected day and time in order to clean chamber trays and refill water bottles. Pellet dispensers were randomly refilled within a 3-day window.

The response measure was the time of occurrence of photobeam breaks in the head trough. The dependent variable was the proportion of response rate. First, an average number of photobeam breaks in the head trough per hour was calculated for the last 10 cycles (i.e., 20 days) of training and for the $96 \mathrm{~h}$ of testing. Next, a response rate measure was calculated by dividing the averaged photobeam breaks during each hour by 60 (i.e., responses/min). Subsequently, a relative measure was calculated in order to account for individual response rates. This was accomplished by summing the response rates across all hours (42 and $96 \mathrm{~h}$ in training and testing, respectively) and then dividing the response rate at each hour by this sum.

\section{RESULTS}

An actogram illustrating the activity of a representative rat is shown in Figure 1. The activity during the meals was concentrated during the first $2 \mathrm{~h}$, followed by intermittent activity throughout the remainder of the meal. As the number of cycles increased, more activity occurred prior to the meal. Activity could also be observed in the middle of the intermeal interval (approximately 21-24 h), although to a lesser extent than before or during the meal.

Figure 2 shows the proportion of response rate during the intermeal interval averaged over the last 10 cycles of training. The proportion of response rate was calculated by dividing the mean response rate during each hour by the sum of the response rates in all $42 \mathrm{~h}$ of the intermeal interval. The proportion of response rate increased immediately before the meal and approximately $24 \mathrm{~h}$ prior to the meal. An ANOVA using proportion of response rate during the intermeal interval as a repeated measure revealed a significant effect of time $[F(41,205)=15.8, p<.05]$. A contrast between the rates at the first and last hours showed that the terminal rate was higher than that observed at the start of the intermeal interval $[F(1,5)=16.8, p<.05]$. To document the existence of the secondary peak near $24 \mathrm{~h}$, the data from hours 15-30 were subjected to a repeated measures ANOVA. This test confirmed a significant effect of time $[F(15,75)=3.1, p<.05]$. To document that the terminal increase in rate was greater than that observed in the secondary peak, we compared the response rate at 21

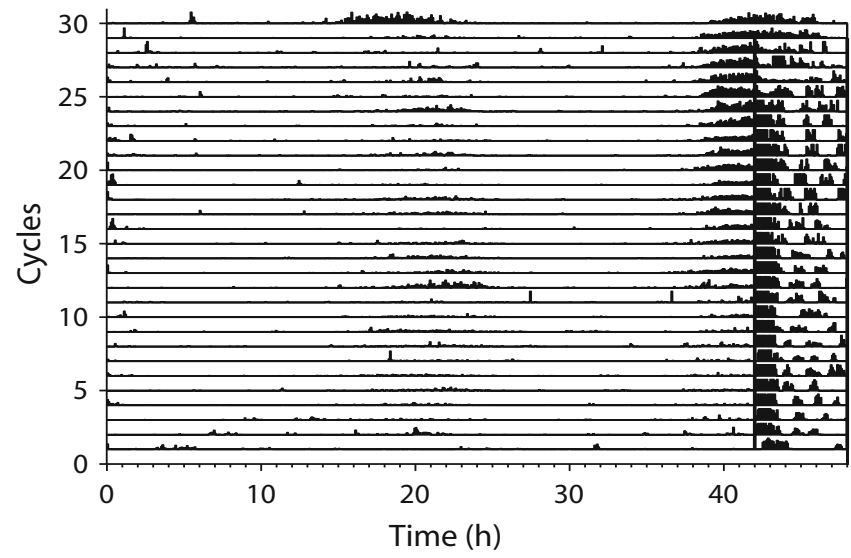

Figure 1. Representative actogram illustrating the behavior of a rat during the study. The $x$-axis plots the time during each cycle, whereas each line along the $y$-axis represents one 48 -h cycle of activity. The data were examined in 5-min bins; the histogram was capped with a maximum value of 300 responses per bin. The vertical lines on the right bracket the time at which food was available.

and $42 \mathrm{~h}$, which revealed a significant difference $[t(5)=$ $6.6, p<.05]$. Lastly, it appears that the shape of the secondary peak may be proportional to the terminal increase. However, because the center of the terminal increase is not known, it is difficult to evaluate the scalar property.

To estimate the location of the secondary peak in Figure 2, a 3-point running median was applied to the averaged training data for each rat, and the time of occurrence of the peak was identified by the local maximum in proportional response rate. The secondary peak (mean $\pm S E$ ) occurred at $22.5 \pm 0.6 \mathrm{~h}$, which was not reliably different from $24 \mathrm{~h}[t(5)=-2.5, p>.05]$.

The proportion of response rate during the 96-h deprivation period is plotted in Figure 3. Note that the measurement of the proportional response rate for the probe differs from that for training. This difference emerges because different response rate sums were used to calculate the proportion of response rate in training and probe conditions. The sum of the response rates in training was for a 42-h period, whereas the probe sum was for a 96-h period. Four peaks appear in Figure 3, with the last three appearing to be relatively large, as compared with the first peak. The proportion of response rate for the 96-h probe was subjected to a repeated measures ANOVA, which revealed a significant effect of time $[F(95,475)=6.9, p<.05]$. The spacing between peaks appears to be approximately $24 \mathrm{~h}$. To estimate the interpeak interval in Figure 3, a 3-point running median was applied to the probe data for each rat, and the time of occurrence of the peak was identified by the local maximum in proportional response rate. The interpeak intervals (mean $\pm S E$ ) were $22.8 \pm 0.5,21.9 \pm$ 2.0 , and $24.4 \pm 1.0 \mathrm{~h}$. In each case, these values were not reliably different from $24 \mathrm{~h}[t(5) \mathrm{s}<2.37, p \mathrm{~s}>.05]$.

Alternation with respect to the status of the meal in the previous day predicts two large peaks in the final $48 \mathrm{~h}$ of the probe. By contrast, a 48-h oscillator mechanism predicts a single large peak at the end of the final $48 \mathrm{~h}$ of the 


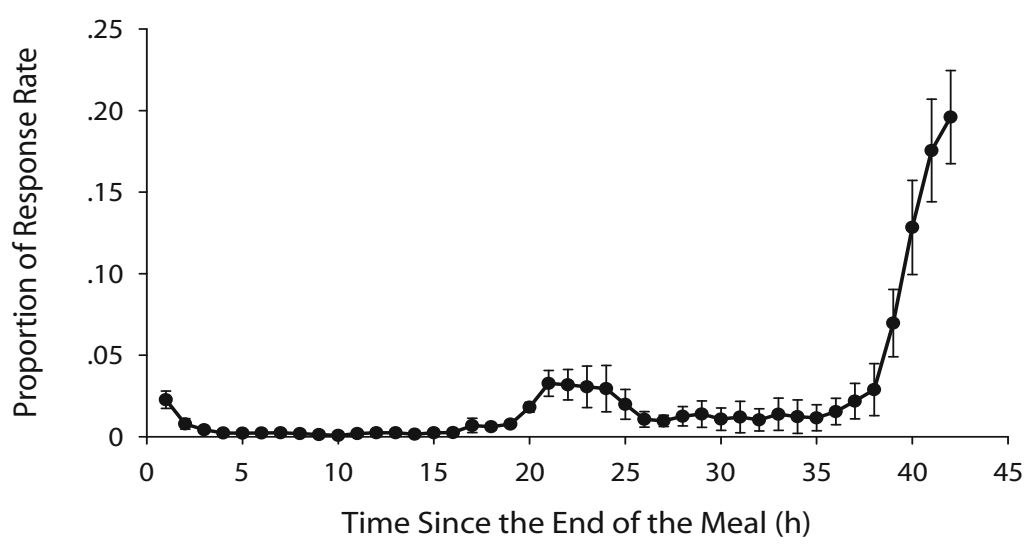

Figure 2. Proportion of response rate as a function of time following the meal during the last 10 cycles of training. Error bars represent the standard error of the mean.

probe (a small peak would also be expected, given the secondary peak documented in Figure 2). Finally, an intervaltiming mechanism does not predict any large peaks in the final $48 \mathrm{~h}$ of the probe. Individual ANOVAs were conducted on each of the peaks separately, using 13-h segments centered on each peak (hours: 15-27, 37-49, 6173, and 84-96). Each of the ANOVAs on data representing the second, third, and fourth peaks revealed a significant effect of time $[F(12,60) \mathrm{s}>2.24, p \mathrm{~s}<.05]$. However, the ANOVA on data representing the first peak did not reach significance $[F(12,60)=1.48, p>.05]$. The inability to detect the first peak in Figure 3 as reliable is likely due to the use of a single cycle in Figure 3, as compared with the documented first peak in Figure 2, which used 10 cycles of data. Note that prior to the absence of food at $42 \mathrm{~h}$ in Figure 3, the procedures were identical in Figures 2 and 3. Therefore, the inability to detect a peak at $24 \mathrm{~h}$ in Figure 3 is likely due to the use of a single cycle.

Next, we sought to characterize the third peak in Figure 3 as large or small. An average response rate was cal- culated for Peaks 1 and 3, using the same data as those used in the analysis above. The proportion of response rate during the third peak was reliably higher than that during the first peak $[t(5)=-2.9, p<.05]$. This is the same conclusion that was reached about Figure 2 when the terminal increase in rate was compared with the rate in the earlier secondary peak.

\section{DISCUSSION}

In this study, we investigated whether rats could learn a 48-h pattern of food availability and evaluated mechanisms potentially involved in this process. Rats lived in an operant chamber for 2 months, during which time food was available every $48 \mathrm{~h}$. There were two increases in responding during training. First, an increase in the response rate was evident at approximately $24 \mathrm{~h}$. Second, a more robust increase in response rate appeared just prior to the meal. At the end of training, the rats were deprived of food in order to observe behavior in the absence of periodic

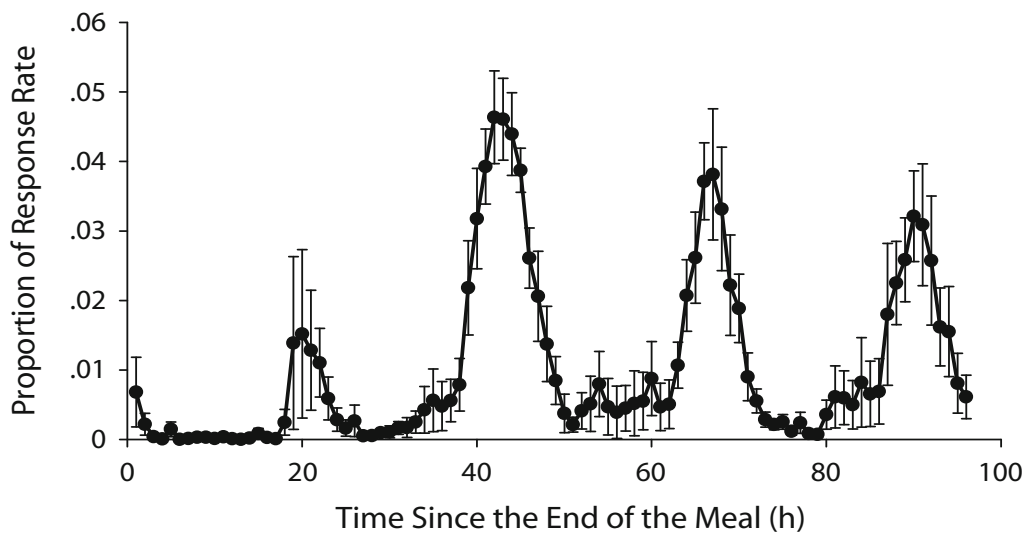

Figure 3. Proportion of response rate as a function of time during the probe (i.e., during extinction). The probe data were collected after the rats had received meals on alternate days. Although predictions of the proposed mechanisms are confounded during training, they are dissociated in the probe (see the text for details). Error bars represent the standard error of the mean. 
input. During this probe, the rats searched the food trough at approximately $24-\mathrm{h}$ intervals.

The present results suggest that the rats were able to anticipate meals separated by 48 -h intermeal intervals. The rats appeared to anticipate the meals on the basis of an alternation mechanism. After a nonfood day, the rats responded at a relatively high rate on the following day. Similarly, after a food day, the rats responded at a relatively low rate on the following day. Importantly, when food was discontinued in the probe, the rats treated each 24-h segment as if it had been preceded by a nonfood day. By contrast, interval timing with respect to the meal predicts that periodic behavior in the probe will cease after the first 48-h interval had elapsed. The last two peaks at the end of the probe suggest that the rats were not timing an interval with respect to the meal. In addition, the data are not compatible with a 48-h endogenous oscillator. The peaks appeared to be spaced every $24 \mathrm{~h}$, with no evidence of a 48 -h period. Unlike a self-sustaining 48 -h oscillator, an alternation mechanism relies upon the status of the event of the previous day, which appears to be the case in the probe condition.

It is possible to conduct further tests of the alternation hypothesis. For example, consider the presentation of an unexpected meal $24 \mathrm{~h}$ after the last meal, followed by omitted meals at 48,72 , and $96 \mathrm{~h}$. According to the alternation hypothesis, the rats would be expected to produce a peak at $72 \mathrm{~h}$ (i.e., $48 \mathrm{~h}$ after the unexpected meal), rather than at $48 \mathrm{~h}$; at subsequent 24-h intervals, additional peaks would be expected according to the alternation hypothesis (i.e., at $96 \mathrm{~h}$ ). By contrast, the interval hypothesis predicts a peak at $72 \mathrm{~h}$ but no additional peak at $96 \mathrm{~h}$. A 48-h selfsustaining oscillator would predict peaks at 48 and $96 \mathrm{~h}$, but not at $72 \mathrm{~h}$.

The method of assessing temporal performance in the present experiment represents an improvement over that used in the unpublished report by Caubisens and Edmonds (Gallistel, 1990). Their previous data, like our data in Figure 2, document discrimination of alternated days without diagnosing the mechanism of temporal discrimination. In particular, there are three mechanisms that could be used to produce the temporal discrimination in Figure 2. The probe was conducted to unconfound the predictions of these mechanisms.

The existence of the initial peak near $24 \mathrm{~h}$ during training complicates the identification of the mechanism responsible for temporal anticipation. Although we have argued that alternation is the likely mechanism responsible for the data observed in the present study, alternation, per se, does not require an increase in response rate at $24 \mathrm{~h}$. It is possible that an error sometimes occurs in the daily conditional discrimination, thereby producing some residual responding at the correct time of day on nonfood days. Another possibility is that in addition to an alternation mechanism, the circadian cue is partially reinforced; in this respect, the circadian cue is unreliable and may result in responding at the correct time of day on both meal and nonmeal days.

The observation that the secondary peak in Figure 2 occurred slightly (but not significantly) before $24 \mathrm{~h}$ may suggest that the rats were responding $24 \mathrm{~h}$ prior to the meal center. In other words, the 6-h meal was noted to occur sometime after $42 \mathrm{~h}$, but not at the 42 nd hour. For example, if the animal recorded that the meal took place at the 45 th hour (i.e., the middle of the meal), responding $24 \mathrm{~h}$ after this would produce an increase in responding just prior to $24 \mathrm{~h}$. Similar reasoning could also explain the location of the first peak in Figure 3. Alternatively, when confronted with the absence of a meal, the rats may have learned to stop responding at the start of the 6-h window in which a meal might occur. This proposal would produce periodic responding that is slightly less than $24 \mathrm{~h}$ during the probe.

In conclusion, rats discriminate alternate days. The observation of temporal discrimination alone is not sufficient to identify the mechanism when multiple cues are confounded. When the multiple cues were dissociated, we found that the rats discriminated alternate days by alternating with respect to the status of the previous day as a meal or a nonmeal day.

\section{AUTHOR NOTE}

These data were presented at the 13th Annual Meeting of the International Conference on Comparative Cognition, Melbourne, FL, 2006. This work was supported by National Institute of Mental Health Grant MH64799 to J.D.C. The raw data (time of occurrence of each stimulus and response) are available at www.uga.edu/animal-cognition-lab. We thank Kenneth W. Maxwell for technical assistance during the course of this study. We also thank Colin Nicholson for his assistance with this study. Correspondence concerning this article should be addressed to J. D. Crystal, Department of Psychology, University of Georgia, Athens, GA 30602-3013 (e-mail: jcrystal@uga.edu).

\section{REFERENCES}

Aragona, B. J., Curtis, J. T., Davidson, A. J., Wang, Z., \& Stephan, F. K. (2002). Behavioral and neurochemical investigation of circadian time-place learning in the rat. Journal of Biological Rhythms, 17, 330-344.

Aschoff, J. (ED.) (1981). Biological rhythms (Handbook of Behavioral Neurobiology, Vol. 4). New York: Plenum.

Bolles, R. C., \& Moot, S. A. (1973). The rat's anticipation of two meals a day. Journal of Comparative \& Physiological Psychology, 83, 510-514.

Boulos, Z., Rosenwasser, A. M., \& Terman, M. (1980). Feeding schedules and the circadian organization of behavior in the rat. $B e-$ havioural Brain Research, 1, 39-66.

CAPAldi, E. J., \& LyNCH, D. (1966). Patterning at 24-hour ITI: Resolution of a discrepancy more apparent than real. Psychonomic Science, 6, 229-230.

Capaldi, E. J., \& Spivey, J. E. (1964). Stimulus consequences of reinforcement and nonreinforcement: Stimulus traces of memory. Psychonomic Science, 1, 403-404.

Church, R. M. (1978). The internal clock. In S. H. Hulse, H. Fowler, \& W. K. Honig (Eds.), Cognitive processes in animal behavior (pp. $277-$ 310). Hillsdale, NJ: Erlbaum.

Crystal, J. D. (2001). Circadian time perception. Journal of Experimental Psychology: Animal Behavior Processes, 27, 68-78.

Crystal, J. D. (2006). Animal behavior: Timing in the wild. Current Biology, 16, R252-R253.

DAAn, S., \& KoEne, P. (1981). On the timing of foraging flights by oystercatchers, Haematopus ostralegus, on tidal mudflats. Netherlands Journal of Sea Research, 15, 1-22.

Escobar, C., Díaz-Muñoz, M., Encinas, F., \& Aguilar-Roblero, R. (1998). Persistence of metabolic rhythmicity during fasting and its entrainment by restricted feeding schedules in rats. American Journal of Physiology, 274, R1309-R1316.

Forel, A. (1910). Das Sinnesleben der Insekten. Munich: Reinhardt. 
Gallistel, C. R. (1990). The organization of learning. Cambridge, MA: MIT Press.

GibBon, J. (1991). Origins of scalar timing. Learning \& Motivation, 22, 3-38.

Gibbon, J., Fairhurst, S., \& Goldberg, B. (1997). Cooperation, conflict and compromise between circadian and interval clocks in pigeons. In C. M. Bradshaw \& E. Szabadi (Eds.), Time and behaviour: Psychological and neurobehavioural analyses (pp. 329-384). New York: Elsevier.

Henderson, J., Hurly, T. A., Bateson, M., \& Healy, S. D. (2006). Timing in free-living rufous hummingbirds, Selasphorus rufus. Current Biology, 16, 512-515.

Mistlberger, R. E., de Groot, M. H. M., Bossert, J. M., \& MarchANT, E. G. (1996). Discrimination of circadian phase in intact and suprachiasmatic nuclei-ablated rats. Brain Research, 739, 12-18.

Pizzo, M. J., \& Crystal, J. D. (2004). Evidence for an alternation strategy in the time-place learning. Behavioural Processes, 67, 533-537.

RichteR, C. P. (1922). A behavioristic study of the activity of the rat. Comparative Psychology Monographs, 1, 1-55.

Rijnsdorp, A., DaAn, S., \& DiJKstra, C. (1981). Hunting in the kes- trel, Falco tinnunculus, and the adaptive significance of daily habits. Oecologia, 50, 391-406.

Silver, R., \& Bittman, E. L. (1984). Reproductive mechanisms: Interaction of circadian and interval timing. In J. Gibbon \& L. Allan (Eds.), Timing and time perception (Annals of the New York Academy of Sciences, Vol. 423, pp. 488-514). New York: New York Academy of Sciences.

Stephan, F. K., Swann, J. M., \& Sisk, C. L. (1979). Anticipation of 24-hr feeding schedules in rats with lesions of the suprachiasmatic nucleus. Behavioral \& Neural Biology, 25, 346-363.

Takahashi, J. S., Turek, F. W., \& Moore, R. Y. (Eds.) (2001). Circadian clocks (Handbook of Behavioral Neurobiology, Vol. 12). New York: Kluwer.

White, W., \& TimberlaKe, W. (1995). Two meals promote entrainment of rat food-anticipatory and rest-activity rhythms. Physiology \& Behavior, 57, 1067-1074.

(Manuscript received December 12, 2006; revision accepted for publication May 10, 2007.) 